Article

\title{
Compact Model for L-Shaped Tunnel Field-Effect Transistor Including the 2D Region
}

\author{
Faraz Najam $(D)$ and Yun Seop Yu *(D) \\ Department of Electrical and Control Engineering and IITC, Hankyong National University, \\ Anseong 17579, Korea \\ * Correspondence: ysyu@hknu.ac.kr; Tel.: +81-31-670-5293
}

Received: 2 August 2019; Accepted: 2 September 2019; Published: 6 September 2019

check for updates

\begin{abstract}
The L-shaped tunneling field-effect transistor (LTFET) is the only line-tunneling type of TFET to be experimentally demonstrated. To date, there is no literature available on the compact model of LTFET. In this paper, a compact model of LTFET is presented. LTFET has both one-dimensional (1D) and 2D band-to-band tunneling (BTBT) components. The 2D BTBT part dominates in the subthreshold region, whereas the 1D BTBT dominates at higher gate-source biases. The model consists of $1 \mathrm{D}$ and 2D BTBT models. The 2D BTBT model is based on the assumption that the electric field originating from the gate and terminating at the source edge is perfectly circular. Tunneling path length is obtained by calculating the distance along an electric field arc that runs from gate to source. The 1D BTBT model is based on a simultaneous solution of the 1D Poisson equation in source and channel regions. Expressions for electric field and potential obtained from integrating the Poisson equation in source and channel regions are solved simultaneously to find the surface potential. Once the surface potential is known, all the other unknown variables, including junction potential and source depletion length, can be calculated. Using the potential profile, tunneling lengths were found for both the source-to-channel BTBT regime, and channel-to-channel BTBT regime. The tunneling lengths were used to calculate the BTBT tunneling rate, and finally, the drain-source current as a function of gate-source, and drain-source bias was calculated. The model results were compared against technology computer-aided design (TCAD) simulation results and were found to be in reasonable agreement for a compact model.
\end{abstract}

Keywords: band-to-band tunneling; L-shaped tunnel field-effect-transistor; 2D BTBT; corner-effect

\section{Introduction}

With the power requirements of complementary metal-oxide-semiconductor (CMOS) technology surging beyond unreasonable levels to meet the high computing demands of today's world, there has been a desperate push for devices that can perform better for less power [1]. The tunnel field-effect transistor (TFET) is one such device among the potential candidate devices [2]. TFET works on the principle of band-to-band-tunneling (BTBT) and achieves a steeper subthreshold slope (SS) than a metal-oxide-semiconductor field-effect transistor (MOSFET) of equivalent dimensions and electrical parameters.

However, the on-current $\left(I_{\mathrm{ON}}\right)$ of TFET is lower than that of MOSFET of equivalent dimensions/electrical parameters. To overcome this problem, different types of TFET architectures have been suggested, including the line-tunneling type [3] TFETs. The structure of line tunneling type TFETs has a gate-source overlap. This overlap increases the BTBT area and consequently increases the $I_{\mathrm{ON}}$. The L-shaped TFET (LTFET) [4] is an example of a line-tunneling TFET that features the channel region grown vertically in the form of an L-shape. The LTFET offers the same benefits as a conventional 
line-tunneling type TFET, but with a much lower device footprint. More significantly, LTFET is the only example of a line-tunneling TFET that has been experimentally realized.

Compact modeling is an important part of the circuit design process. While there are compact models available for the conventional TFET [5-7], the literature is almost completely lacking in compact models for the line-tunneling type TFETs. Vandenberghe et al. [3] developed a compact model for conventional line-tunneling TFET. Najam et al. [8] developed a compact model for LTFET. However, the line-tunneling TFET considered in [3] features a significant difference from the LTFET: The gate directly overlaps with the source region, as shown in Figure 1 in [3], whereas in the LTFET, there is a channel region present between the source and the gate. Direct overlapping of gate/source without any channel layer present in between completely changes the electrostatics of the device and makes the model presented in [3] inapplicable to LTFET. In [8], only the one-dimensional (1D) model is presented. In LTFET, both 1D and 2D BTBT components are present.

This paper presents a complete model of the LTFET, including both the 2D and 1D BTBT, which is presented in Section 2. The model presented is continuous from the subthreshold region to strong inversion. The model is tested for LTFETs with varying geometries, and the results are presented in Section 3. A conclusion is presented in Section 4.

\section{Model Development}

Figure 1 shows the schematic of the LTFET. The channel region shown in blue color is found in an L-shape and is sandwiched between the gate and the source. The part of the channel region found in between the source and gate regions is termed as $C_{\text {overlap }}$ with height $\left(H_{\text {overlap }}\right)=40 \mathrm{~nm}$ and length $\left(T_{\mathrm{j}}\right)=4 \mathrm{~nm}$. The source and drain are $\mathrm{p}^{+}\left(N_{\mathrm{a}}=10^{20} \mathrm{~cm}^{-3}\right)$ and $\mathrm{n}^{+}\left(N_{\text {drain }}=10^{20} \mathrm{~cm}^{-3}\right)$ doped, respectively, while the channel is lightly $\mathrm{n}^{-}$doped $\left(N_{\mathrm{d}}=10^{15} \mathrm{~cm}^{-3}\right)$. The source region height $\left(H_{\mathrm{s}}\right)$ and length $\left(L_{\mathrm{s}}\right)$ are 40 and $50 \mathrm{~nm}$, respectively. The bottom part of the channel, which is not in between the source and gate regions, is termed as $C_{\text {nonoverlap }}$ and has a height $\left(H_{\text {nonoverlap }}\right)=20 \mathrm{~nm}$, and length $\left(L_{\text {nonoverlap }}\right)=50 \mathrm{~nm}$. An $\mathrm{HfO}_{2}$ dielectric of thickness $\left(t_{\mathrm{ox}}\right)=2 \mathrm{~nm}$ for gate oxide was considered.

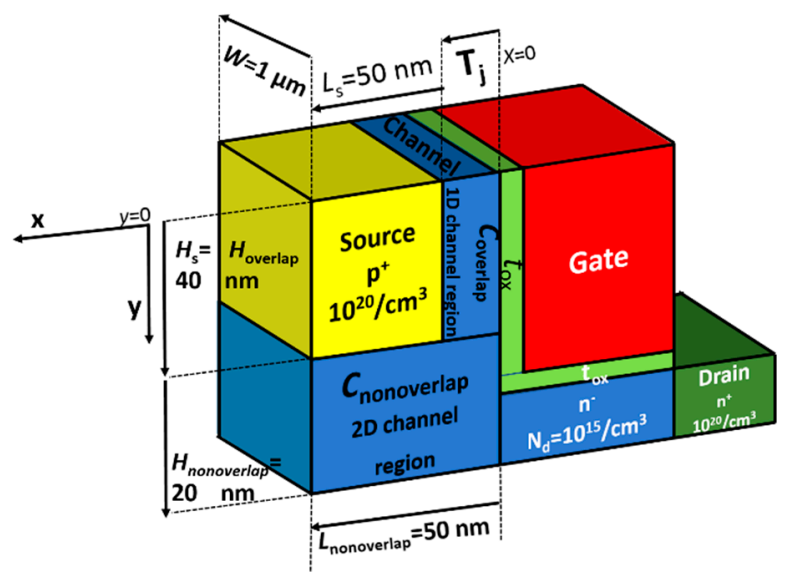

Figure 1. Schematic of the L-shaped tunneling field-effect transistor (LTFET).

Dynamic nonlocal BTBT model [9], fermi statistics, and constant mobility models were considered in the technology computer-aided design (TCAD) simulation.

As shown in Figure 1, the source has a sharp corner marked by an X in Figure 1. The electric field from the gate converges at and around this sharp source corner, increasing the potential around this point. The right axis in Figure 2a shows surface potential $\left(\varphi_{\mathrm{s}}\right)$ at $V_{\mathrm{gs}}=0.15 \mathrm{~V}$ and $V_{\mathrm{ds}}=0.5$ V. As shown in Figure $2 \mathrm{a}, \varphi_{\mathrm{s}}$ sharply rises in $C_{\text {nonoverlap }}$ because of convergence of the electric field, whereas $\varphi_{\mathrm{s}}$ is less in $C_{\text {overlap }}$ where this convergence does not take place. This convergence affects the BTBT threshold voltage of $C_{\text {overlap }}$ and $C_{\text {nonoverlap }}$. $C_{\text {nonoverlap }}$ is found to have a lower BTBT threshold voltage because of this increased $\varphi_{\mathrm{s}}$. Meanwhile, $C_{\text {overlap }}$ has a significantly higher BTBT threshold 
voltage because of the lower potential [10]. This can be seen in Figure $2 b$, which shows integrated BTBT tunneling rates [10] $\left(G_{\text {tun }} \mathrm{s}\right)$ in $x$ and $y$ directions in $C_{\text {overlap }}$ and $C_{\text {nonoverlap, }}$ respectively; that is, $\int_{L_{s}+T_{j}}^{0} \int_{0}^{H_{\text {overlap }}} G_{\text {tun }} d x d y$ in $C_{\text {overlap }}$ (black triangles) and $\int_{L_{\text {nonoverlap }}}^{0} \int_{H_{\text {overlap }}}^{H_{\text {nonovlap }}} G_{\text {tun }} d x d y$ in $C_{\text {nonoverlap }}$ (red circles) in the left axis. Figure $2 \mathrm{~b}$ clearly shows that $C_{\text {nonoverlap }}$ turns on earlier than $C_{\text {overlap }}$. The right axis in Figure $2 \mathrm{~b}$ shows the drain-source current $\left(I_{\mathrm{ds}}\right)$ as a function of gate-source bias $\left(V_{\mathrm{gs}}\right)$ of LTFET. It is shown that in the subthreshold part of the $I_{\mathrm{ds}}-V_{\mathrm{gs}}$ characteristics, only the $C_{\text {nonoverlap }}$ is active. $C_{\text {overlap }}$ turns on at $V_{\mathrm{gs}}=0.15 \mathrm{~V}$. When $C_{\text {overlap }}$ turns on, its $G_{\text {tun }}$ is significantly higher because of $1 \mathrm{D}$ BTBT paths. As a result, it dominates the 2D $G_{\text {tun }}$ in $C_{\text {nonoverlap, }}$ as can be seen in Figure $2 \mathrm{~b}$. Based on this analysis, $I_{\mathrm{ds}}-V_{\mathrm{gs}}$ characteristics of LTFET were modeled in two parts, first the 2D model in $C_{\text {nonoverlap }}$ for the subthreshold region and then the $1 \mathrm{D}$ model in $C_{\text {overlap. }}$.

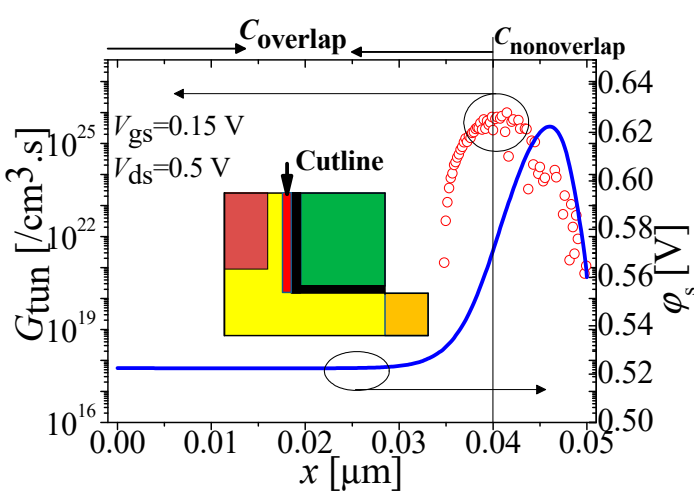

(a)

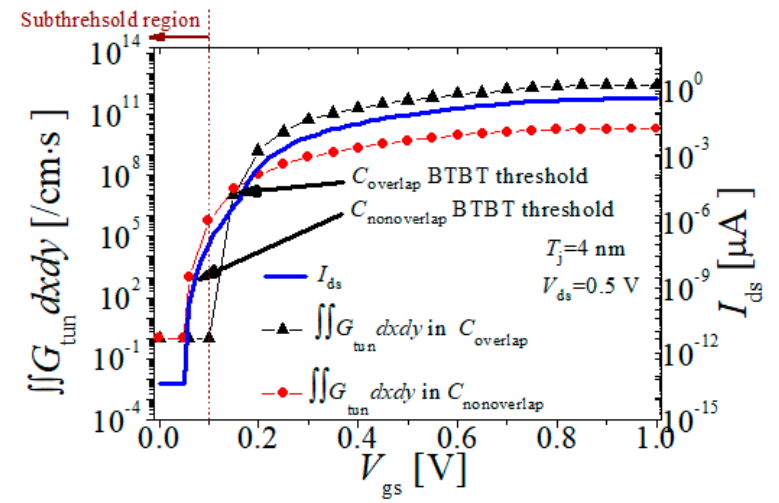

(b)

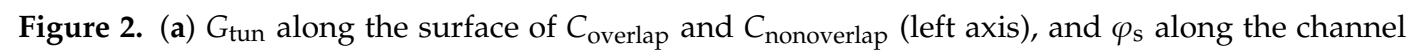
(right axis) at $V_{\mathrm{gs}}=0.15 \mathrm{~V}$ and $V_{\mathrm{ds}}=0.5 \mathrm{~V}$. (b) Integrated band-to-band tunneling (BTBT) tunneling

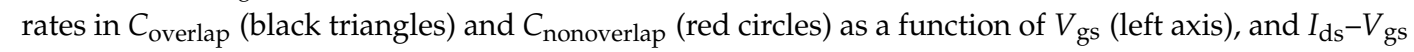
characteristics of LTFET (right-axis). BTBT threshold voltages for $C_{\text {overlap }}$ and $C_{\text {nonoverlap }}$ are indicated by arrows.

\subsection{D Model: $C_{\text {nonoverlap }}$ Model}

The 2D model is based on the work in [3]. The following assumptions are used in this model: (1) Electric fields are assumed to be completely circular and terminate from gate to source. This helps in obtaining a convenient expression for the tunneling length $\left(W_{\mathrm{t}}\right) ;(2)$ Gate dielectric is treated as the same material as the channel with an equivalent dielectric thickness $t^{\prime}{ }_{\mathrm{ox}}$ given by $t_{\mathrm{ox}}=t_{\mathrm{ox}} \varepsilon_{\mathrm{si}} / \varepsilon_{\mathrm{ox}}$, where $t_{\mathrm{ox}}, \varepsilon_{\mathrm{si}}$, and $\varepsilon_{\mathrm{ox}}$ are the physical dielectric thickness, silicon dielectric permittivity, and oxide dielectric permittivity, respectively. This is necessary to ensure a continuous and perfectly circular electric field from the gate-to-channel/gate dielectric interface, and finally terminate at the source. Without this assumption, the electric field will be discontinuous, that is, not perfectly circular in its path from gate to source. In this work, $W_{t}$ is conveniently calculated as the length along the perfectly circular electric field arc, from gate to source, as will be shown below. If a discontinuity arises in the circularity of the electric field arc, such convenient calculation of $W_{t}$ will not be possible; (3) The source is assumed to be completely depleted, and depletion length is ignored. The source is heavily doped. Depletion length is inversely proportional to doping concentration [3]. This makes the source depletion length negligible. Including the source depletion length would add complexity to the model without significantly increasing the accuracy of the model; (4) The source is assumed to be touching the gate. Table 1 mentions most of the symbols used in the equations below.

With these assumptions, the boundary conditions at source and gate can be given by

$$
\varphi(x, 0)=\varphi_{\text {source }} \text { and } \varphi(0, y)=\varphi_{\mathrm{g}} .
$$


where $\varphi_{\text {source }}$ is assumed to be $0 \mathrm{~V}$ and $\varphi_{\mathrm{g}}$ is the gate-source bias minus the flat band voltage $\left(V_{\mathrm{fb}}\right)$, that is, $\varphi_{\mathrm{g}}=V_{\mathrm{gs}}-V_{\mathrm{fb}}$. The solution of potential in polar co-ordinates is given by

$$
\varphi(x, y)=\frac{2 \theta \varphi_{\mathrm{g}}}{\pi}, \quad 0 \leq \theta \leq \frac{\pi}{2} .
$$

The above boundary condition along with $\theta_{0}$ is shown in Figure $3 \mathrm{a}$. Thanks to assumptions 1 and $2, W_{t}$ is calculated as the length along a perfectly circular electric field line as follows:

$$
W_{\mathrm{t}}=r_{0} \theta_{0}
$$

where $\theta_{0}$ is given by $\theta_{0}=\pi E_{\mathrm{g}} /\left(2 q \varphi_{\mathrm{g}}\right)$, (where $E_{\mathrm{g}}$ is the bandgap, and $q$ is the charge on an electron) and is the angle when the potential difference between the source edge and some point along the electric field line becomes equal to $E_{\mathrm{g}} / q$. $\theta_{0}$ is obtained by substituting the BTBT condition, that is, $\varphi=E_{\mathrm{g}} / q$ in (2), and inverting it. Since $\theta_{0}$ is bias-dependent, $\theta_{0}$ decreases, and $W_{\mathrm{t}}$ decreases as $V_{\mathrm{gs}}$ bias increases. This is illustrated in Figure $3 \mathrm{~b} . r_{0}$ is the radius of the electric field arc and is given by $r_{0}=t^{\prime}{ }_{\text {ox }} / \cos \left(\theta_{0}\right)$. Drain current expression in $C_{\text {nonoverlap }}\left(I_{\mathrm{ds} \_}\right.$Cnonoverlap $)$is given by the following equation for $D=2.5[3]$ :

$$
I_{\mathrm{ds}_{-} \text {Cnonoverlap }}=\frac{q W A_{\mathrm{k}} E_{\mathrm{g}} t_{\mathrm{ox}}^{\prime}}{q^{4.5} B_{\mathrm{k}}^{2}} \cdot \frac{1}{\theta_{0}^{4.5} r_{0}^{3.5}} \cdot \exp \left(-q B_{\mathrm{k}} r_{0} \theta_{0} \sqrt{E_{\mathrm{g}}}\right),
$$

where $W\left(=10^{-4} \mathrm{~cm}\right)$ is the device width, and $A_{\mathrm{k}}=1 \times 10^{15} \mathrm{eV}^{0.5} \cdot \mathrm{cm}^{-1 / 2} \cdot \mathrm{s}^{-1} \cdot \mathrm{V}^{-2.5}$ and $B_{\mathrm{k}}=1.5 \times 10^{7}$ $\mathrm{V} \cdot \mathrm{cm}^{-1} \cdot \mathrm{eV}^{-1.5}$ are the parameters used in the dynamic nonlocal BTBT model. There is one notable difference between this work and [3] which is that, as $C_{\text {overlap }}$ turns on, $I_{\mathrm{ds} \_ \text {Cnonoverlap }}$ is assumed to saturate. This is in line with the results presented in Figure $2 \mathrm{~b}$. Once $C_{\text {overlap }}$ turns on, it dominates, and $C_{\text {nonoverlap }}$ does not have any significant contribution beyond that point. Without this assumption, the model overestimates $I_{\mathrm{ds} \_ \text {Cnonoverlap }}$ in the high $V_{\mathrm{gs}}$ region.

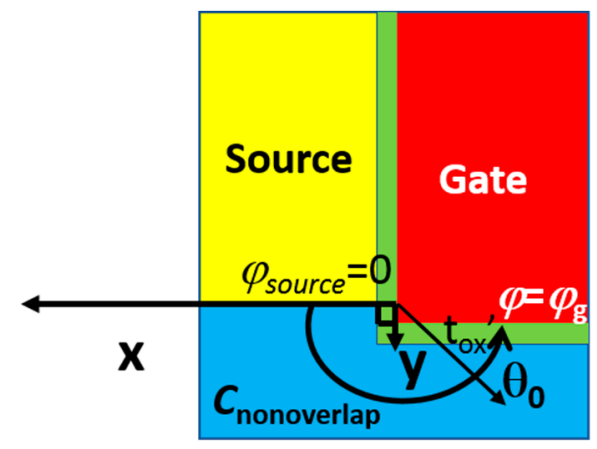

(a)

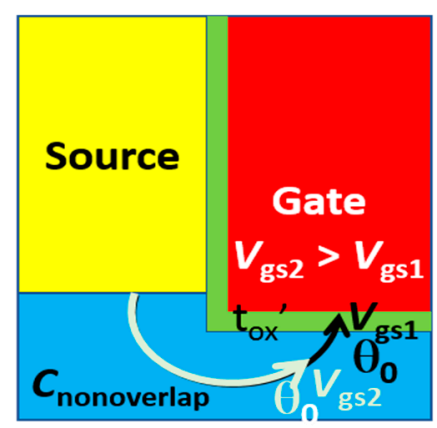

(b)

Figure 3. (a) Schematic illustrating the boundary conditions and the assumptions used in the model. (b) Schematic showing $\theta_{0}$ at a lower $V_{\mathrm{gs}}$ bias, $V_{\mathrm{gs} 1}$ (black), and at a higher $V_{\mathrm{gs}}$ bias, $V_{\mathrm{gs} 2}$ (white).

Table 1. List of all the symbols specific to the two-dimensional (2D) model.

\begin{tabular}{ccc}
\hline Symbol & Description & Value/Unit \\
\hline$\varphi_{\text {source }}$ & Potential at source edge & $\mathrm{V}$ \\
$\varphi_{\mathrm{g}}$ & Potential at gate & $\mathrm{V}$ \\
$t_{\mathrm{ox}}$ & Physical oxide thickness & $2 \times 10^{-7} \mathrm{~cm}$ \\
$t_{\mathrm{ox}}^{\prime}$ & Equivalent semiconductor thickness & $\mathrm{cm}$ \\
$\varepsilon_{\mathrm{si}}, \varepsilon_{\mathrm{ox}}$ & Silicon, oxide permittivity & $11.9,25 \mathrm{~F} / \mathrm{cm}$ \\
$\theta_{0}$ & Angle when the BTBT condition is satisfied & Radian \\
$r_{0}$ & Radius of an electric field arc & $\mathrm{cm} / \mathrm{radian}$ \\
\hline
\end{tabular}




\subsection{D Model: $C_{\text {overlap }}$ Model}

Figure 4 a shows a magnified $C_{\text {overlap }}$ and source regions. The channel region considered in the $1 \mathrm{D}$ model comprises the $C_{\text {overlap }}$ region. Figure 4 a mentions the important parameters used in the 1D model, including the location of $\varphi_{\mathrm{s}}$ and junction potential $\left(\varphi_{\mathrm{j}}\right) \cdot \varphi_{\mathrm{j}}$ is the potential at the junction of the source and channel region in Figure 4a. The device origin is at the top of the channel/gate dielectric interface, and $x_{\text {channel }}$ and $x_{\text {source }}$ correspond to the $x$-coordinate in channel and source regions, respectively. The dimension for the $1 \mathrm{D}$ model is along the $x$-direction, as shown by the cutline shown in Figure $4 \mathrm{~b}$. The cutline begins at the channel/gate dielectric interface and ends in the source region. Figures $5 d-f$ and $6 a, b$ are along the black cutline shown in Figure $4 b$.

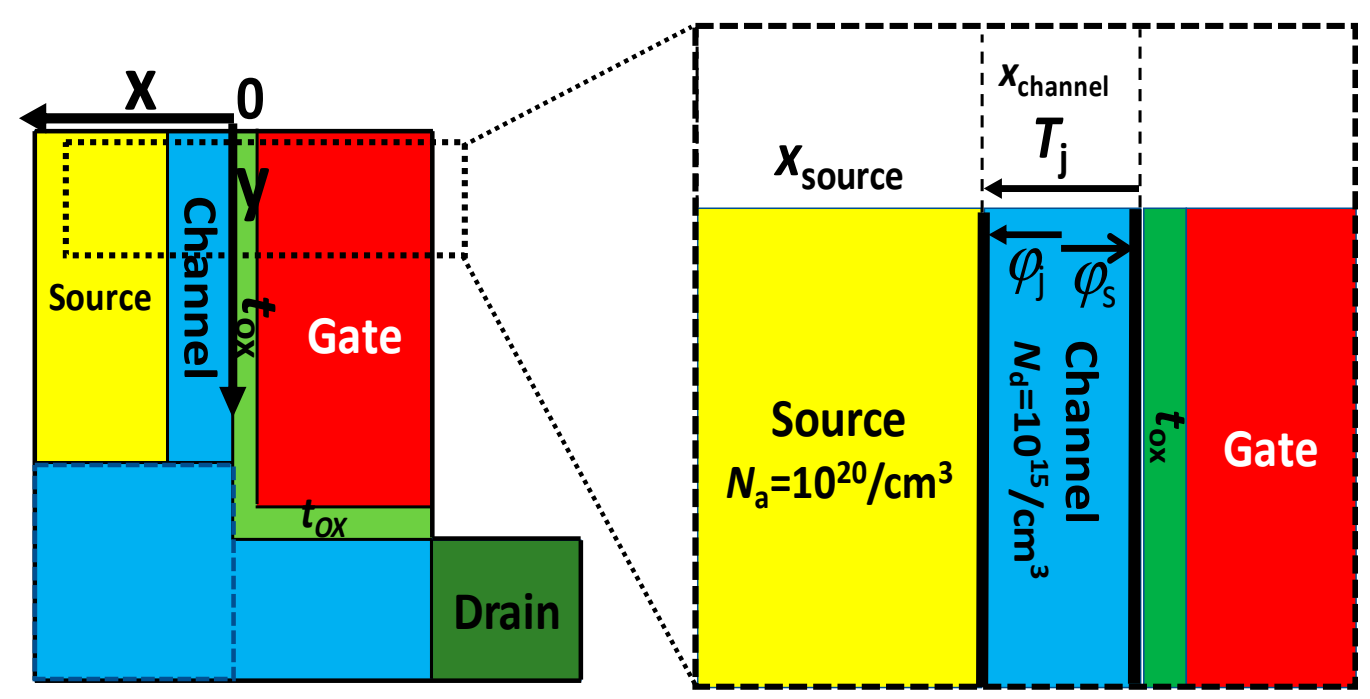

(a)

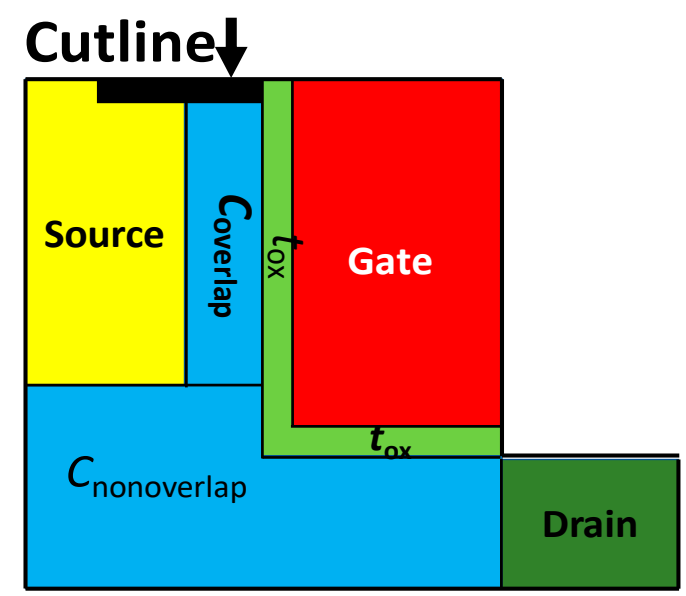

(b)

Figure 4. (a) Magnified part of $C_{\text {overlap }}$ and source regions of the LTFET to illustrate parameters used in the 1D model. Device origin is at the dielectric/channel interface. $x$ and $y$ are in the horizontal and vertical directions, respectively. (b) Only a single $y$-point is considered in the 1D model, illustrated by the cutline. Figures $5 \mathrm{~d}-\mathrm{f}$ and $6 \mathrm{a}, \mathrm{b}$ to follow are along the same cutline with $x=0 \mathrm{~nm}$ at the channel-dielectric interface. 


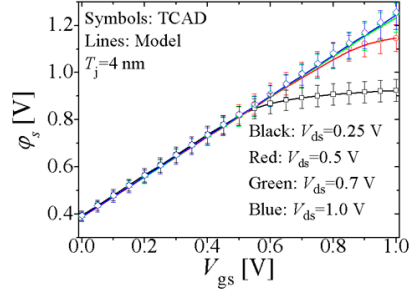

(a)

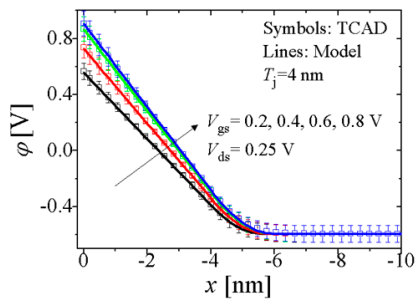

(d)

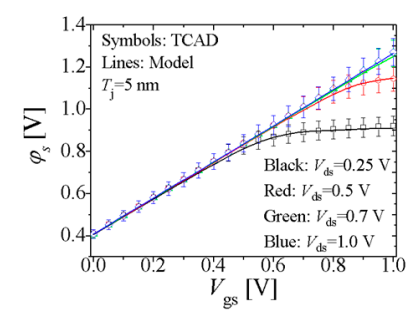

(b)

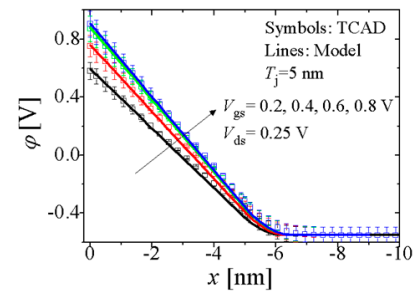

(e)

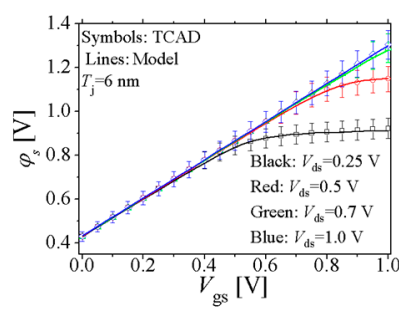

(c)

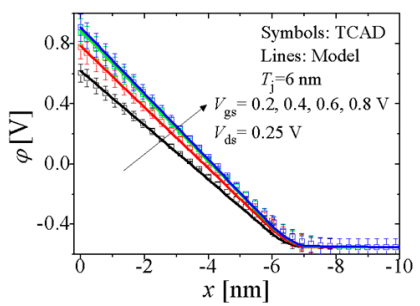

$(\mathbf{f})$

Figure 5. (a-c) $\varphi_{\mathrm{s}}$ as a function of $V_{\mathrm{gs}}$, for $T_{\mathrm{j}}=4,5$, and $6 \mathrm{~nm}$, respectively, at $V_{\mathrm{ds}}=0.25$ (black), 0.5 (red), 0.7 (green) nm, and $1 \mathrm{~V}$ (blue). Error bar: $5 \%$. (d-f) potential profile in channel, and source regions, for $T_{\mathrm{j}}=4,5$, and $6 \mathrm{~nm}$, respectively, at $V_{\mathrm{gs}}=0.2$ (black), 0.4 (red), 0.6 (green), and $0.8 \mathrm{~V}$ (blue) at $V_{\mathrm{ds}}=0.25 \mathrm{~V}$. Error bar: $10 \%$. Lines: Potential model. Symbols: technology computer-aided design (TCAD). Figure $5 d-f$ is along the cutline of Figure $4 b$.

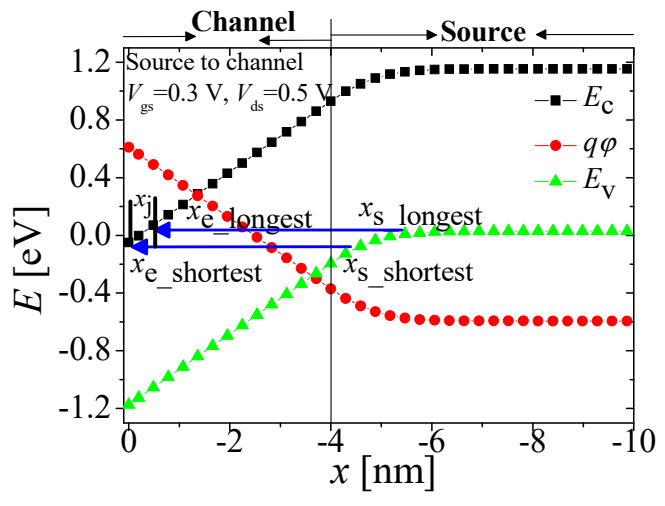

(a)

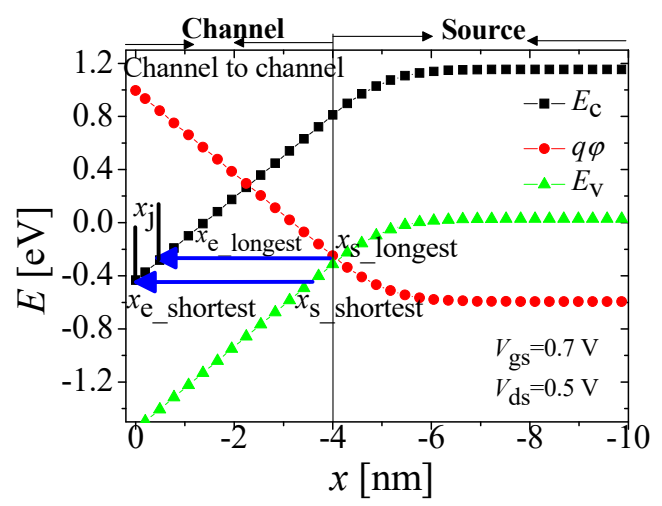

(b)

Figure 6. (a,b) Band diagram at $V_{\mathrm{gs}}=0.3$ and $0.7 \mathrm{~V}$, respectively, and both at $V_{\mathrm{ds}}=0.5 \mathrm{~V}$, along the cutline of Figure 4 . Black squares, green triangles, and red circles represent $E_{\mathrm{c}}, E_{\mathrm{V}}$, and $q \varphi$. respectively. Source-to-channel (a) and channel-to-channel (b) BTBT mechanisms are indicated with the help of arrows.

The 1D model is based on the solution of the 1D Poisson equation. Integrating the 1D Poisson equation once, in source and channel regions, and neglecting electron and hole carrier concentrations yields the electric field in the respective regions, which are given by

$$
\begin{gathered}
\frac{\partial \varphi_{\text {source }}}{\partial x_{\text {source }}}=\frac{q N_{\mathrm{a}}}{\varepsilon_{\mathrm{si}}} x_{\text {source }}+\frac{q N_{\mathrm{a}}}{\varepsilon_{\mathrm{si}}}\left(L_{\mathrm{dep}}\right), \\
\frac{\partial \varphi_{\text {channel }}}{\partial x_{\text {channel }}}=\frac{q N_{\mathrm{d}}}{\varepsilon_{\mathrm{si}}} x_{\text {channel }}+\frac{\varepsilon_{\mathrm{ox}}}{\varepsilon_{\mathrm{si}} t_{\mathrm{ox}}}\left(V_{\mathrm{gs}}-V_{\mathrm{fb}}-\varphi_{\mathrm{s}}\right),
\end{gathered}
$$


where $L_{\mathrm{dep}}$ is the depletion length of the source region. Integrating (5) and (6) again yields potential in source and channel regions, which is given by

$$
\begin{gathered}
\varphi_{\text {source }}\left(x_{\text {source }}\right)=\frac{q N_{\mathrm{a}}}{2 \varepsilon_{\mathrm{si}}}\left(x_{\text {source }}+L_{\mathrm{dep}}\right)^{2}+\varphi_{\text {dep }} \\
\varphi_{\text {channel }}\left(x_{\text {channel }}\right)=\frac{q N_{\mathrm{d}}}{2 \varepsilon_{\mathrm{si}}} x_{\text {channel }}^{2}+\frac{\varepsilon_{\mathrm{ox}}}{\varepsilon_{\mathrm{si}} t_{\mathrm{ox}}}\left(V_{\mathrm{gs}}-V_{\mathrm{fb}}-\varphi_{\mathrm{s}}\right) x_{\text {channel }}+\varphi_{\mathrm{s}} \\
\varphi_{\mathrm{s}}=V_{\mathrm{gs}}-V_{\mathrm{fb}}+\frac{q\left(N_{\mathrm{a}}+N_{\mathrm{d}}\right)}{C_{\mathrm{ox}}} T_{\mathrm{j}}+\frac{q \varepsilon_{\mathrm{si}} N_{\mathrm{a}}}{C_{\mathrm{ox}}^{2}} \\
-\gamma \sqrt{V_{\mathrm{gs}}-V_{\mathrm{fb}}+\frac{q\left(N_{\mathrm{a}}+N_{\mathrm{d}}\right)}{2 \varepsilon_{\mathrm{si}}} T_{\mathrm{j}}^{2}+\frac{q\left(N_{\mathrm{a}}+N_{\mathrm{d}}\right)}{C_{\mathrm{ox}}} T_{\mathrm{j}}+\frac{q \varepsilon_{\mathrm{si}} N_{\mathrm{a}}}{2 \mathrm{C}_{\mathrm{ox}}^{2}}-\varphi_{\mathrm{dep}}}
\end{gathered}
$$

where $C_{\mathrm{ox}}$ is the gate oxide capacitance, $\varphi_{\mathrm{dep}}$ is the source depletion potential, and $\gamma=\left(2 \varepsilon_{\mathrm{si}} q N_{\mathrm{a}}\right)^{1 / 2} / C_{\mathrm{ox}}$. With the potential profile known, $W_{t}$ can be calculated. Because (9) is derived from the depletion approximation, the smoothing function from [11-13] was used to model strong inversion of the electron.

There are two different 1D BTBT mechanisms present in LTFET [8]. One is the source-to-channel BTBT, which starts at low bias, and the other is the channel-to-channel BTBT, which takes place at high $V_{\text {gs }}$ bias. The first source to channel the 1D BTBT model is discussed.

The potential profile within the channel is assumed to be linear, as seen in Figure $5 d-f$, which is given by

$$
\varphi_{\text {channel }}\left(x_{\text {channel }}\right)=m x_{\text {channel }}+\varphi_{\mathrm{s}}
$$

where $m$ is the slope of the linear potential profile in the channel, $m=\left(\varphi_{\mathrm{s}}-\varphi_{\mathrm{j}}\right) / T_{\mathrm{j}} . \varphi_{\mathrm{j}}$ can be found from (8) by using $x_{\text {channel }}=T_{\mathrm{j}}$. Figure 5a-c shows $\varphi_{\mathrm{s}}$ as a function of $V_{\mathrm{gs}}$ of LTFET with $T_{\mathrm{j}}=4$, 5 , and $6 \mathrm{~nm}$ at different $V_{\mathrm{ds}}$ biases, respectively. Figure $5 \mathrm{~d}-\mathrm{f}$ shows the potential profile along the cutline shown in Figure $4 \mathrm{~b}$ for LTFET with $T_{\mathrm{j}}=4,5$, and $6 \mathrm{~nm}$ at different $V_{\mathrm{gs}}$ and $V_{\mathrm{ds}}=0.25 \mathrm{~V}$, respectively. Symbols and lines denote the simulation results of TCAD and the proposed potential model, respectively. Reasonable agreement is observed within a maximum error of $10 \%$ between the model and TCAD simulations.

Figure 6a shows a band diagram at $V_{\mathrm{gs}}=0.3 \mathrm{~V}$ and $V_{\mathrm{ds}}=0.5 \mathrm{~V}$, along the cutline of Figure $4 \mathrm{~b}$. Black and green symbols represent conduction band minimum energy $\left(E_{\mathrm{c}}\right)$ and valence band maximum energy $\left(E_{\mathrm{v}}\right)$, respectively. Red circles represent potential. Arrows denote $W_{\mathrm{t}} \mathrm{s}$. The longest $W_{\mathrm{t}}\left(W_{\mathrm{t} \_l o n g e s t}\right)$ originates where $\varphi_{\text {source }}=\varphi_{\text {dep }}$, and the shortest $W_{\mathrm{t}}\left(W_{\mathrm{t} \_ \text {shortest }}\right)$ originates from where the potential is the highest, that is, $\varphi_{\mathrm{s}}$. The starting and ending points for $W_{\mathrm{t} \_ \text {shortest }}$ are $x_{\mathrm{s}_{s} \text { shortest }}$ and $x_{\mathrm{e}_{\_} s h o r t e s t}$, respectively, and the starting and ending points for $W_{\mathrm{t}_{\_} \text {longest }}$ are $x_{\mathrm{s}_{-} \text {longest }}$ and $x_{\mathrm{e} \_ \text {longest }}$, respectively, which are all indicated by arrows in Figure 6a. $x_{\mathrm{s}_{-} \text {longest }}$ naturally starts from $L_{\mathrm{dep}}$, that is, $x_{\mathrm{s}_{-} \text {longest }}=$ $-a b s\left(L_{\mathrm{dep}}+T_{\mathrm{j}}\right)$, and the ending point for the $W_{\mathrm{t} \_ \text {shortest }}$ is the surface, that is, $x_{\mathrm{e} \_ \text {shortest }}=0 . x_{\mathrm{e} \_l o n g e s t}$ is the point where the BTBT condition for $W_{\mathrm{t}_{\_} \text {longest }}$, that is, $\varphi\left(x_{\mathrm{e} \_ \text {longest }}\right)=\varphi_{\text {dep }}+E_{\mathrm{g}} / q$, is satisfied. By substituting this BTBT condition in (10) and inverting it, $x_{\mathrm{e}_{-} \text {longest }}$ is given by $x_{\mathrm{e} \_l \text { longest }}=\left(\varphi_{\mathrm{dep}}+E_{\mathrm{g}} / q-\right.$ $\left.\varphi_{\mathrm{s}}\right) \mathrm{T}_{\mathrm{j}} /\left(\varphi_{\mathrm{s}}-\varphi_{\mathrm{j}}\right) \cdot x_{\mathrm{s}_{\mathrm{s}} \text { shortest }}$ is the point where the BTBT condition for $W_{\mathrm{t} \_ \text {shortest }}$, that is, $\varphi\left(x_{\mathrm{s}_{-} \text {shortest }}\right)=\varphi_{\mathrm{s}}$ $-E_{\mathrm{g}} / q$, is satisfied. By substituting this BTBT condition in (7) and inverting it, $x_{\mathrm{s}_{-} \text {shortest }}$ is given by

$$
x_{\mathrm{s} \_ \text {shortest }}=\sqrt{\varphi_{\text {dep }}+\varphi_{\mathrm{s}}-\frac{E_{\mathrm{g}}}{q}\left(\frac{2 \varepsilon_{\mathrm{si}}}{q N_{\mathrm{a}}}\right)}-a b s\left(L_{\mathrm{dep}}+T_{\mathrm{j}}\right)
$$

Finally, $W_{\mathrm{t}_{\_} \text {shortest }}$ and $W_{\mathrm{t} \_ \text {longest }}$ are given by

$$
\begin{gathered}
W_{\mathrm{t} \_ \text {shortest }}=x_{\mathrm{e} \_ \text {shortest }}-x_{\mathrm{s} \_ \text {shortest }} \\
W_{\mathrm{t} \_ \text {longest }}=x_{\mathrm{e} \_ \text {longest }}-x_{\mathrm{s} \_ \text {longest }}
\end{gathered}
$$


$G_{\text {tun }}$ is given by Kane's model [14]

$$
G_{\mathrm{tun}}=\frac{A_{\mathrm{k}}}{\sqrt{E_{\mathrm{g}}}}\left(\frac{E_{\mathrm{g}}}{q W_{\mathrm{t}}}\right)^{2.5} \exp \left(-q B_{\mathrm{k}} W_{\mathrm{t}} \sqrt{E_{\mathrm{g}}}\right) .
$$

$I_{\mathrm{ds}}$ for source-to-channel BTBT $\left(I_{\mathrm{ds}_{-} \_\_}\right)$is given by

$$
I_{\text {ds_s_c }}=q W \int_{0}^{H_{\mathrm{s}}} \int_{0}^{x_{\mathrm{j}}} G_{\text {tun }}(x) d y d x=q W H_{\mathrm{s}} x_{\mathrm{j}}\left(\frac{G_{\text {tun_shortest }}+G_{\text {tun_longest }}}{2}\right)
$$

where $x_{\mathrm{j}}$ is the integration limit indicated in Figure 6a, and is equal to $x_{\mathrm{j}}=x_{\mathrm{e} \_ \text {shortest }}-x_{\mathrm{e} \_l \text { longest }}$. In (14), a constant average $G_{\text {tun }}$, that is, $\frac{G_{\text {tun_shortest }}+G_{\text {tun_longest }}}{2}$, is used. Here, $G_{\text {tun_shortest }}$ and $G_{\text {tun_longest }}$ are found by using $W_{\mathrm{t}}=W_{\mathrm{t} \_ \text {shortest }}$ and $W_{\mathrm{t} \_ \text {longest }}$ in (13), respectively. $G_{\text {tun }}$ is a function of $W_{\mathrm{t}}$, as can be inferred from (13). The integral in (14), however, is with respect to $x$. Finding a closed-form expression for $I_{\mathrm{ds} \_} s_{-} \mathrm{c}$ then necessitates expressing $W_{\mathrm{t}}$ as a function of $x$. However, because $W_{\mathrm{t}}$ cannot be expressed as a function of $x, W_{t}$ can only be found for fixed BTBT boundary conditions, as done in (12a, b). In this scenario, $d W_{\mathrm{t}} / d x$ cannot be evaluated. As a result, there is no closed-form expression available for $G_{\text {tun }}$ integrated as a function of $x$. Therefore, the simplification of using average $G_{\text {tun }}$ was necessary and, as it will be shown in Section 3, the average $G_{\text {tun }}$ approximates the integral of $G_{\text {tun }}$ with respect to $x$ reasonably well. When the bias is high enough, the potential increases so much that BTBT becomes possible from even inside the channel. This is illustrated by the band diagram shown in Figure $6 \mathrm{~b}$ along the cutline of Figure $4 \mathrm{~b}$. Here, $E_{\mathrm{v}} / E_{\mathrm{c}}$ becomes aligned within the channel, as illustrated by the arrows, in addition to the source/channel $E_{\mathrm{v}} / E_{\mathrm{c}}$ alignment. Here, $W_{\mathrm{t} \_l o n g e s t}$ starts from $x_{\mathrm{s}_{\_} \text {longest }}=T_{\mathrm{j}}$ and ends at $x_{\mathrm{e} \_l o n g e s t}$, where the BTBT condition, $\varphi\left(x_{\mathrm{e} \_ \text {longest }}\right)=\varphi_{\mathrm{j}}+E_{\mathrm{g}} / q$, is satisfied. Similarly, $W_{\mathrm{t} \_ \text {shortest }}$ starts at $x_{\mathrm{S}_{s} \text { shortest }}$, where the BTBT condition, $\varphi\left(x_{\mathrm{s}_{-} \text {shortest }}\right)=\varphi_{\mathrm{s}}-E_{\mathrm{g}} / q$, is satisfied and ends at $x_{\mathrm{e}_{-} \text {shortest }}=0$. By substituting these boundary conditions in (10) and inverting it, $x_{\text {e_longest }}$ and $x_{\mathrm{s}_{-} \text {shortest }}$ can be calculated as $x_{\mathrm{e}_{-} \text {longest }}=\left(\varphi_{\mathrm{j}}+E_{\mathrm{g}} / q-\varphi_{\mathrm{s}}\right) T_{\mathrm{j}} /\left(\varphi_{\mathrm{s}}-\varphi_{\mathrm{j}}\right)$ and $x_{\mathrm{s}_{-} \text {shortest }}=-E_{\mathrm{g}} T_{\mathrm{j}} /\left(q\left(\varphi_{\mathrm{s}}-\varphi_{\mathrm{j}}\right)\right)$. As can be seen in Figure $6 \mathrm{~b}, W_{\mathrm{t}}$ is almost constant within the channel. Therefore, in the channel-to-channel regime, $G_{\text {tun_shortest }} \approx G_{\text {tun }}\left(W_{\mathrm{t}_{\text {_longest }}}\right) \approx G_{\text {tun }}\left(W_{\mathrm{t}_{\text {_shortest }}}\right)$. This means that $G_{\text {tun }}(x)$ can be taken out of the integral in the channel-to-channel drain current $\left(I_{\text {ds_c_c }}\right)$ expression, which is given by

$$
I_{\mathrm{ds} \_\mathrm{c} \_\mathrm{c}}=q W H_{\mathrm{s}} x_{\mathrm{j}} G_{\text {tun }} \approx q W H_{\mathrm{s}} x_{\mathrm{e} \_ \text {longest }} G_{\text {tun_shortest }}
$$

The total $I_{\mathrm{ds}}$ is given by

$$
I_{\mathrm{ds}}=I_{\mathrm{ds} \_ \text {Cnonoverlap }}+I_{\mathrm{ds} \_ \text {s_c }}+I_{\mathrm{ds} \_\mathrm{c} \_\mathrm{c}}
$$

\section{Results}

Figure $7 \mathrm{a}-\mathrm{c}$ shows $I_{\mathrm{ds}}-V_{\mathrm{gs}}$ characteristics of LTFET with $T_{\mathrm{j}}=4,5$, and $6 \mathrm{~nm}$, respectively. Symbols and lines denote the simulation results of TCAD and the proposed model, respectively. Blue, red, and black colors denote $V_{\mathrm{ds}}=0.25,0.5$, and $0.7 \mathrm{~V}$, respectively. A kink is observed in Figure 7a-c, at the transition point where the 1D model takes over the 2D model. This is observed because the 1D and 2D models are independent of each other and don't produce the same and continuous $G_{\text {tun }}$ at the transition point. 


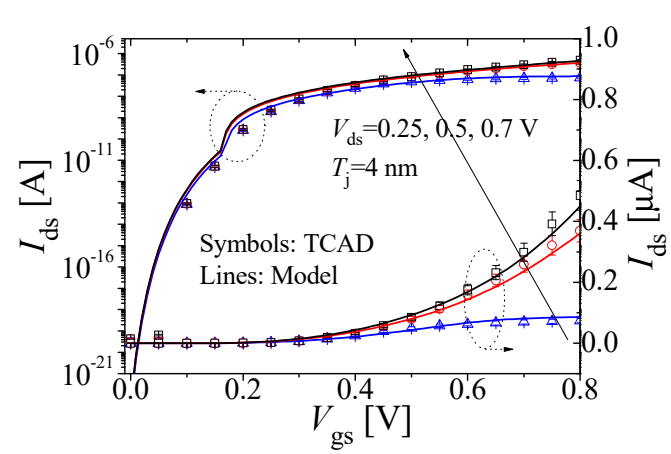

(a)

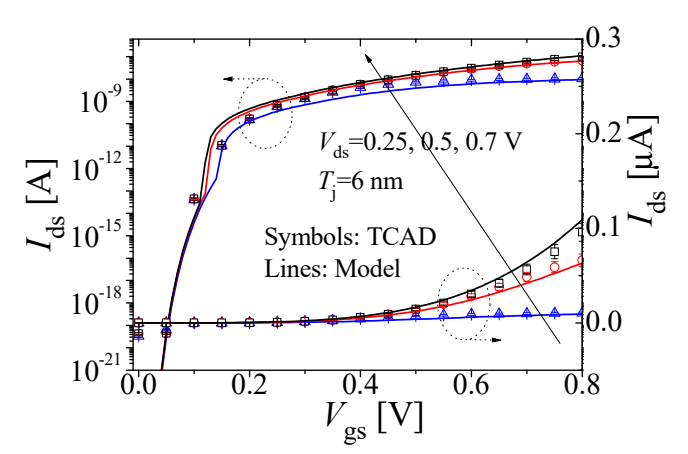

(c)

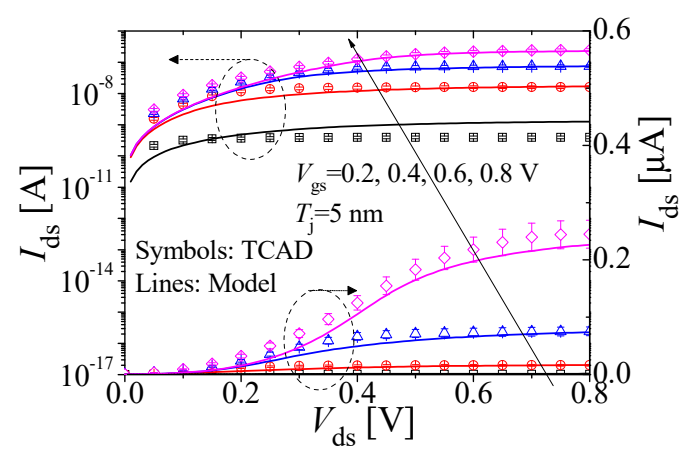

(e)

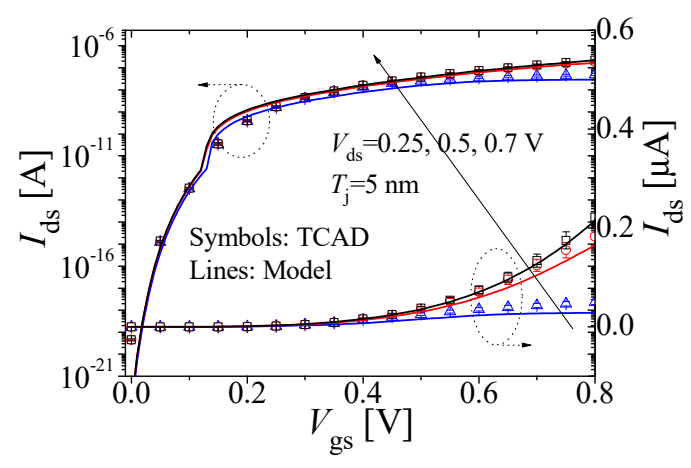

(b)

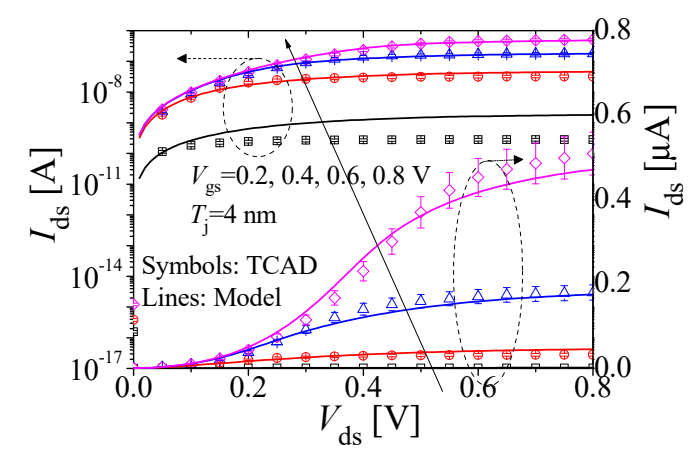

(d)

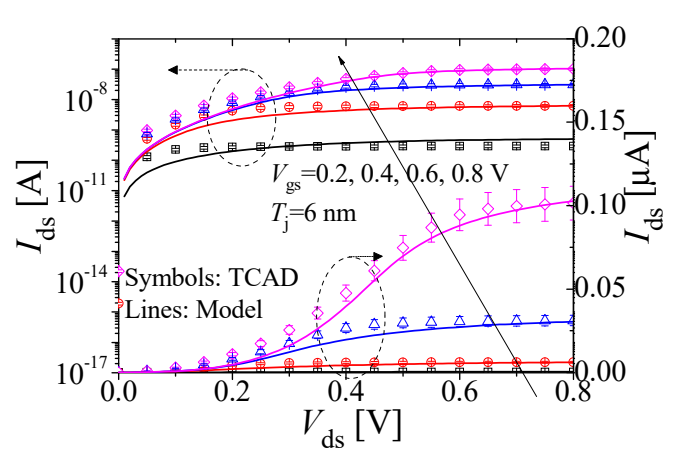

(f)

Figure 7. (a-c) $I_{\mathrm{ds}}-V_{\mathrm{gs}}$ characteristics of LTFET with $T_{\mathrm{j}}=4,5$, and $6 \mathrm{~nm}$, respectively, at $V_{\mathrm{ds}}=0.25$ (blue), 0.5 (red), and $0.7 \mathrm{~V}$ (black). (d-f) $I_{\mathrm{ds}}-V_{\mathrm{ds}}$ characteristics of LTFET with $T_{\mathrm{j}}=4,5$, and $6 \mathrm{~nm}$, respectively, at $V_{\text {gs }}=0.2$ (black), 0.4 (red), 0.6 (blue), and $0.8 \mathrm{~V}$ (magenta). Error bar: $10 \%$. Lines: Model. Symbols: TCAD.

There is no noticeable change observed in the subthreshold behavior as $T_{\mathrm{j}}$ is changed from $4 \mathrm{~nm}$ to $6 \mathrm{~nm}$. However, the on-current $\left(I_{\mathrm{ON}}\right)$ is observed to decrease as $T_{\mathrm{j}}$ is increased. $I_{\mathrm{ON}}$ at $V_{\mathrm{gs}}=0.8 \mathrm{~V}$ and $V_{\mathrm{ds}}=0.7 \mathrm{~V}$ is $0.48,0.22$, and $0.09 \mu \mathrm{A}$ for $T_{\mathrm{j}}=4,5$, and $6 \mathrm{~nm}$, respectively. This is because $W_{\mathrm{t}}$ and $G_{\text {tun }}$ are inversely proportional, as can be observed from (13); the longer $W_{t}$ in $T_{j}=5$ and $6 \mathrm{~nm}$ results in lower $G_{\text {tun }}$, and consequently, lower $I_{\mathrm{ds}}$. This effect is captured by the model reasonably well. This suggests that shorter $T_{\mathrm{j}}$ is more desirable for getting higher $I_{\mathrm{ON}}$.

Figure $7 \mathrm{~d}-\mathrm{f}$ shows $I_{\mathrm{ds}}-V_{\mathrm{ds}}$ characteristics of the LTFET for $T_{\mathrm{j}}=4,5$, and $6 \mathrm{~nm}$, respectively. Symbols denote TCAD simulation results, and lines denote model results. Black, red, blue, and magenta denote $V_{\mathrm{gs}}=0.2,0.4,0.6$, and $0.8 \mathrm{~V}$, respectively. As shown in Figure $7 \mathrm{~d}-\mathrm{f}$, the saturation characteristics of the LTFET are predicted reasonably well by the model. 
Figure 8 shows $I_{\mathrm{ds}}-V_{\mathrm{gs}}$ characteristics of LTFET with $T_{\mathrm{j}}=4 \mathrm{~nm}$ and $H_{\text {overlap }}=H_{\mathrm{s}}$ varied at $V_{\mathrm{ds}}=0.5 \mathrm{~V}$. Symbols denote TCAD simulation results and lines denote model results. Blue, red, and black represent $H_{\text {overlap }}=40,50$, and $60 \mathrm{~nm}$, respectively. It is shown in Figure 8 that, as $H_{\text {overlap }}=H_{\mathrm{s}}$ is increased, the $I_{\mathrm{ds}}$ increases. This is because, with an increase in $H_{\text {overlap }}$, the BTBT area increases. This results in an increase in $I_{\mathrm{ds}}$. Once again, the model captures this effect reasonably well.

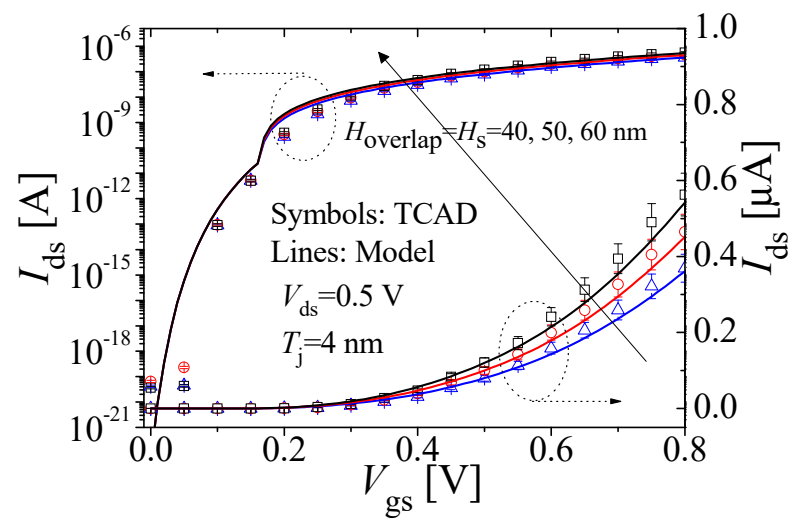

Figure 8. $I_{\mathrm{ds}}-V_{\mathrm{gs}}$ characteristics of LTFET with $T_{\mathrm{j}}=4 \mathrm{~nm}, H_{\text {overlap }}$ varied, and $V_{\mathrm{ds}}=0.5 \mathrm{~V}$. Blue, red, and black represent $H_{\text {overlap }}=H_{\mathrm{s}}=40,50$, and $60 \mathrm{~nm}$, respectively. Symbols and lines denote TCAD simulation and model results, respectively. Error bar: $10 \%$.

Compared to the planar TFET, the LTFET offers better SS and $I_{\mathrm{ON}}$ performance. This can be gauged from the fact that while in planar TFET, the dominant BTBT generation area comprises the surface source/channel depletion regions. In the LTFET, however, because of the gate-source overlap, this area is significantly amplified by the height of the source and $C_{\text {overlap }}$ regions. Furthermore, because of this overlap, the electrostatic coupling between gate and source is stronger in LTFET. In other words, the electric field is stronger in the LTFET as compared to the planar TFET, which also results in higher $I_{\mathrm{ON}}$ and lower inverse subthreshold slope in LTFET, as was demonstrated even in the case of the experimental LTFET and planar TFET in [4].

For a compact model, the simulation results of the model agree reasonably well with those of TCAD within a maximum error of $10 \%$. However, it is not entirely accurate. The inaccuracy results from the simplified integral expression in (14). Numerical integration of $G_{\text {tun }}$ with respect to $x$ will significantly improve the result. Another source of error is the use of the smoothing function to model electron inversion. The smoothing function only approximates surface potential saturation due to electron inversion, and is thus not very accurate at high bias. Considering the electron concentration term in the Poisson equation and doing a self-consistent solution for potential and electron concentration will also improve the model accuracy significantly. However, both numerical integration and self-consistent potential electron concentration solution will significantly add to the computational complexity of the model. This will make the model unsuitable for SPICE applications.

It should also be mentioned that the model was bench-marked only against TCAD data and not experimental data. This is because the experimental LTFET demonstrated significant trap-assisted tunneling (TAT), and Shockley-Read-Hall (SRH) generation-recombination current- and ambipolar current-induced degradation of the $I_{\mathrm{ds}}-V_{\mathrm{gs}}$ characteristics [4]. In particular, TAT in line TFETs is a significant topic and requires its own modeling framework [9]. This work with the equations for surface and junction potentials lays the foundation for the TAT model. However, due to space constraints, TAT, SRH, ambipolar, quantum confinement [15], and breakdown models [16] could not be included in this manuscript. 


\section{Conclusions}

A compact model for LTFET was presented. The model calculates both the 1D BTBT and 2D BTBT present in LTFET. The 1D model is based on the simultaneous solution of 1D Poisson equations in the channel and source region. The Poisson equation is integrated twice in both regions, and the expression for electric field and potential are equated at the source-channel junction point to yield expression for surface potential. To model electron inversion, a simple smoothing function was used. After obtaining the potential profile, starting and ending points of tunneling paths were determined using BTBT boundary conditions. The shortest tunneling path was considered in the drain current expression for source-to-channel BTBT. This was done to obtain a simplified expression for the source-to-channel drain current. Tunneling path lengths were similarly determined for the channel-to-channel BTBT regime. The model was compared against $I_{\mathrm{ds}}-V_{\mathrm{gs}} / V_{\mathrm{ds}}$ results obtained from the simulator for different $V_{\mathrm{ds}} / V_{\mathrm{gs}}$ biases, and for different channel region thicknesses, and heights. The results of the compact model are in reasonable agreement with the simulator results.

Author Contributions: Conceptualization, F.N. and Y.S.Y.; methodology, F.N. and Y.S.Y.; investigation, F.N. and Y.S.Y.; data curation, F.N.; writing-original draft preparation, F.N.; writing-review and editing, F.N. and Y.S.Y.; supervision, Y.S.Y.; project administration, Y.S.Y.; funding acquisition, Y.S.Y.

Funding: This research was funded by the Ministry of Trade, Industry, and Energy (MOTIE), project number 10054888 and Korea Semiconductor Research Consortium (KSRC) support program for the development of future semiconductor devices.

Acknowledgments: This work was supported by IDEC (EDA tool).

Conflicts of Interest: The authors declare no conflict of interest.

\section{References}

1. International Roadmap for Devices and Systems (IRDS). 2017. Available online: https://irds.iee.org/editions/ 2017 (accessed on 5 September 2019).

2. Avci, U.E.; Morris, D.H.; Young, I.A. Tunnel field-effect-transistors: Prospects and challenges. IEEE J. Electron Devices Soc. 2015, 3, 88-95. [CrossRef]

3. Vandenberghe, W.G.; Verhulst, A.S.; Groeseneken, G.; Soree, B.; Magnus, W. Analytical model for point and line tunneling in a tunnel field-effect transistor. In Proceedings of the 2008 International Conference on Simulation of Semiconductors Processes and Devices, Hakone, Japan, 9-11 September 2008.

4. Kim, S.W.; Kim, J.H.; Liu, T.K.; Choi, W.Y.; Park, B. Demonstration of L-shaped tunnel field-effect transistors. IEEE Trans. Electron Devices 2016, 63, 1774-1778. [CrossRef]

5. Yu, Y.S.; Najam, F. Compact current model of single-gate/double-gate tunneling field-effect transistors. J. Electr. Eng. Technol. 2017, 12, 2014-2020. [CrossRef]

6. Zhang, L.; He, J.; Chan, M. A compact model for double-gate tunneling field-effect-transistors and its implications on circuit behaviors. In Proceedings of the 2012 International Electron Devices Meeting, San Francisco, CA, USA, 10-13 December 2012.

7. Wan, J.; Royer, C.L.; Zaslavsky, A.; Cristoloveanu, S. A tunneling field effect transistor model combining interband tunneling with channel transport. J. Appl. Phys. 2011, 110. [CrossRef]

8. Najam, F.; Yu, Y.S. Investigation of corner effect and identification of tunneling regimes in L-shaped tunnel field-effect-transistor. J. Nanosci. Nanotechnol. 2018, 18, 6578-6583. [CrossRef]

9. Synopsys. User Manual, Version L-2016.03, Synopsys TCAD Sentaurus; Synopsys: San Jose, CA, USA, 2016.

10. Najam, F.; Yu, Y.S. Impact of quantum confinement on band-to-band-tunneling of line-tunneling type L-shaped tunnel field-effect-transistor. IEEE Trans. Electron Devices 2019, 66, 2010-2016. [CrossRef]

11. Yu, Y.S.; Kim, N.H. One dimensional analytical model of L-shaped tunnel field-effect-transistor. In Proceedings of the 12th International Conference on Future Information and Communication Engineering, Sapporo, Japan, 25-28 June 2019.

12. Wu, C.; Huang, R.; Huang, Q.; Wang, C.; Wang, J.; Wang, Y. An analytical surface potential model accounting for the dual-modulation effects in tunnel FETs. IEEE Trans. Electron Devices. 2014, 61, 2690-2696. [CrossRef] 
13. Zhang, L.; Chan, M. SPICE modeling of double-gate tunnel-FETs including channel transports. IEEE Trans. Electron Devices 2014, 61, 300-306. [CrossRef]

14. Kane, O.E. Theory of tunneling. J. Appl. Phys. 1961, 32, 83-91. [CrossRef]

15. Walke, A.M.; Verhulst, A.S.; Vandooren, A.; Verreck, D.; Simoen, E.; Rao, V.R.; Groeseneken, G.; Collaert, N.; Thean, A.V.Y. Part I: Impact of field-induced quantum confinement on the subthreshold swing behavior of line TFETs. IEEE Trans. Electron Devices 2013, 60, 4057-4064. [CrossRef]

16. Jung, H.; Dimitrijev, S. Analysis of flat-band-voltage dependent breakdown voltage for $10 \mathrm{~nm}$ double gate MOSFET. J. Inf. Commun. Converg. Eng. 2018, 16, 43-47. [CrossRef] 